\title{
Development of an IoT Based Shopping Trolley
}

\author{
Namratha.G \\ CSE,GAT \\ Bangalore \\ namrathaganesh090@gmail.com
}

\author{
Akhila Srinath \\ CSE,GAT \\ Bangalore \\ akhilasrinath@gmail.com
}

\author{
Snigdha Sen \\ CSE,GAT \\ Bangalore \\ snigdha.sen@gat.ac.in
}

\author{
Abhilash.N \\ CSE,GAT \\ Bangalore \\ abhilash.nataraj@gat.ac.in
}

\author{
Ashuthosh.M.G \\ CSE,GAT \\ Bangalore \\ ashumg760@gmail.com
}

\begin{abstract}
The modern technology has augmented the quality of living for the humans. There has been an emerging demand for fast and straightforward payment of bills in supermarkets. People crave for a high quality with modern technology in everything we tend to use in our daily lives. This project describes the way to build an automatic and time saving system for the globe of retail which can build shopping experience impetuous, client friendly and secure. In the reality, shopping at super markets, malls has resulted in massive crowds to long lines at the billing counter as a result of the cashier has got to scan each product item then enter it into the billing record. The existing billing system may be a bit time overwhelming and that's where the thought of IoT based Automated Shopping Trolley -a remedial electronic product to catch-up with this downside emerged. This is supported by Raspberry Pi fitted with a LED screen and QR scanner and a wireless technology referred to as Bluetooth. The digital display(LED) used may be a 16x2 and Bluetooth modules build the wireless network to figure simply between a particular ranges. A unique product with social acceptance is that the one that aids the comfort, convenience and potency in standard of living. The temporary description of its operation is, after you choose a product and drop it into the trolley, the QR scanner scans the product's distinctive code and its worth.. Card payment facility is also made available below the LCD display. If payment is via cash then he/she has to visitthecounterandpaythebillasdisplayedontheLCDscreenfit.Thiswillsavethetimethat was earlier being consumed to scan each item. The entire system will work on RFID tags. The entire database and product info is stored using Cloud Technology. Anti-theft protection is enabled. Indoor Navigation facility is also enabled.
\end{abstract}

Keywords- LCD, QR Scanner,Raspberry Pi, RFID,Cloud Technology, Anti-theft, IoT.

$* * * * *$

\section{INTRODUCTION}

Humans nowadays invariably select the technology that is helpful to them and have always fictitious a technology which can support their wants. Basically, human desires to decrease the tasks using the technology in quicker and easier approach in varied fields obtainable. A main thing where human spend most time is shopping. according to survey we can say human spend approximately 1 to 1.5 hours for shopping and most of the shoppers can invariably tend to steer out of a queue if it's long. As we all know there are two forms of shopping i.e. 1) shopping nose to nose, 2) on-line shopping, here on-line shopping is that the easiest method to buy as we don't need to be present physically within the shop or mall. wherever shopping nose to nose need to visit the mall or buy shopping where client have to wait within the queue for a while.

In nowadays, in each supermarkets and malls have shopping trolley and baskets for patrons to store the purchased merchandise. once shopping is finished customers need to proceed to checkout at the asking counter. Here this asking method is sort of time intense and need to use additional human resource at the billing section. thus in this paper 'Automated shopping Trolley' which can cut back the time of shoppers and can decrease the manpower at the asking section and increase potency. in the world where technology is very important and the future of retail trade is additionally lies in additional machine-controlled devices..

\section{DEFINITIONS}

IoT: The Internet of Things (IoT) is a system of physical things embedded with sensors, software, electronics and connectivity to allow it to perform better by exchanging information with other connected devices, the operator or the manufacturer.

System Design: System design is the process of defining the elements of a system such as the architecture, modules and components, the different interfaces of those components and the data that goes through that system. 


\section{REVIEW OF LITERATURE}

A. System Study

Literature Survey is an important phase in the system development life cycle as it collects and acquires the necessary information to handle or develop a project during this phase. A literature review is a description of the literature relevant to particular field or topic. It gives an overview of what has been said, who the key writers are, what are the prevailing theories and hypothesis and what methods and what methodologies are appropriate and useful.

In this section, research is done prior to taking up the project and understanding the various methods that were used previously. A detailed analysis of the existing systems was performed. This study helped to identify the benefits and drawbacks of the existing systems.

Since this project itself is an application based on RFID Technology, the literature survey has been done on some of the papers regarding components and some methodologies or techniques. Based on the requirement of the project information has been collected from these papers.

\section{B. Literature Review}

[1] Supermarket is a place where people come to purchase their daily using products and pay for that. So there is a need to calculate the total products and total amount. Here the use of RFID tags to decrease the time and reduce labor costs by shifting it to self-service. The use of Zigbee technology reduces low data rate, low cost and low power consumption.

[2] This paper is proposed to develop a system that is able to scan dynamic and static products in the shopping space using RFID Reader antennas. Instead of conducting the RFID observations at the level of individual carts, aislelevel scanning is performed.

[3] In this paper each and every product had RFID tag instead of barcode scanner. The smart trolley will contain RFID reader, LCD display and Zigbee transmitter. When a person puts a product in the trolley it will scan the product and it's cost which will be displayed on the LCD. RFID (radio frequency identification) automatically identifies and track tags attached to the objects.

\section{A.}

\section{PROPOSED WORK}

\section{Problem Statement.}

Design a system which will reduce billing time in the supermarket and will be cost efficient. An innovative product with societal acceptance is the one that aids the comfort, convenience and efficiency in everyday life. Purchasing and shopping at big malls is becoming daily activity in metro cities. People purchase different items and put them in the trolley. After completion of purchases, one needs to go to billing counter for payments. At billing counter the cashier prepares the bill using bar code reader which is very time consuming process and results in long queue at billing counter. Instead of wasting time standing and waiting for individual turns, one can utilize such a time in a productive way.

Another problem faced by most of the people is about locating the products they are in need. Also most of the individuals are finding difficulties in knowing about the prevailing offers available for a particular product. Another major concern for shopkeepers is that people might take away or steal the products which is also another loss.

\section{B. Targeted work for Problem Statement}

In this Project, a system is implemented which is being developed to assist a person in everyday shopping in terms of reduced time spent while purchasing. The main objective of proposed system is to provide a technology oriented, low-cost, easily scalable, and rugged system for assisting shopping in person.

In this project, the other idea which is being implemented is data analysis. As the customer starts putting things in the trolley, the machine recognizes the customers interest and will suggest the customers about similar offers. There is an indoor navigation facility available to search the products inputted to the list. Addressing the last issue, Anti-theft protection is also enabled.

\section{Existing System}

- Manual billing

- Use Barcode for billing

- Human staff is needed for billing

\section{Scope of the Project}

The basis of accomplishment lies in understanding the innovation and different components to minimize the potential issues. It is time for the Industry to begin using the new innovation, for example, RFID in different applications. In this project the items in the cart can be restricted because customers don't prefer excess luggage at once. This project reduces and eliminates the time taken in billing counter in super markets by designing an Intelligent Shopping Basket which uses Barcode scanners to allow users to self-checkout and increase productivity time. Taking into account the changing trend in retail shopping, the Intelligent Shopping Basket project is most certainly a definite necessity for the Retail marketing industry to step up their portfolios, cope up with the advancement in technology and save time and manpower. 


\section{SYSTEM REQUIREMENTS AND} SPECIFICATIONS.

\section{A. Functional Requirements}

The functional requirements for a system describe what the system should do. These requirements depend on the type of software being developed, the general approach taken by the organization when writing requirements. The functional system requirements describe the system function in detail, its inputs and outputs, exceptions and so on.

Functional requirements are as follows:

- Capacity -The system is enough capable to hold the data and process on it.

- Activity - For each activity, the data will be recorded in the application.

- Interface Requirement - The system is capable to accept and transmit the raw data which may be in the form of digital that is numeric values.

\section{B. Hardware Requirements}

- Power Supply: In most of our electronic products or projects we need a power supply for converting mains AC voltage to a regulated DC voltage. The blocks for designing a power supply are transformer, bridge rectifier, filter, and regulator. In this project, a dual power supply one of $5 \mathrm{~V}$ and one of $12 \mathrm{~V}$ is used and also a step down transformer which converts $230 \mathrm{~V} 50 \mathrm{~Hz}$ to a required small voltage is also used.

- SD Card: Raspberry PI has no storage on board. $\mathrm{SD} /$ micro SD Card stores the OS. The total memory of the SD card is about $8 \mathrm{~GB}$.

- Network Adapter: Wireless network connection is used for the process of data transfer. Wi-Fi USB Adapter that supports speed of $150 \mathrm{Mbps}$ is used. Edimax is a commonly used Wi-Fi USB Adapter.

- LCD Display : The most commonly used LCDs found in the market today are 1 Line, 2 Line or 4 Line LCDs which have only 1 controller and support at most of 80 characters, whereas LCDs supporting more than 80 characters make use of 2 HD44780 controllers. Most LCDs with 1 controller has 14 Pins and LCDs with 2 controller has 16 Pins (two pins are extra in both for back-light LED connections).

- QR Scanner: QR code (abbreviated from Quick Response Code) is the trademark for a type of matrix barcode (or two-dimensional barcode) first designed for the automotive industry in Japan. A barcode is a machine-readable optical label that contains information about the item to which it is attached. A QR code uses four standardized encoding modes (numeric, alphanumeric, byte/binary, and kanji) to efficiently store data; extensions may also be used.
- Raspberry Pi: The Raspberry Pi is designed to be connected to the Internet. Its ability to communicate on the Internet is one of its key features and opens up all sorts of possible uses, including home automation, web serving, network monitoring, and so on. The connection can be wired through an Ethernet cable (at least in the case of the model B), or the Pi can use a USB WiFi module to provide a network connection. This is very useful in situations where the Raspberry Pi itself is in- accessible and does not have a keyboard, mouse, and monitor attached to it.

- Camera: The Raspberry Pi Camera Module is a custom designed add-on for Raspberry Pi. It attaches to Raspberry Pi by way of one of the two small sockets on the board upper surface. This interface uses the dedicated CSI interface, which was designed especially for interfacing to cameras. CSI bus is capable of extremely high data rates, and it exclusively carries pixel data

C. Software Requirements

- OS installation : At first, install the OS and software to the Raspberry Pi before mounting it all together. An OS is the basic operating system software that tells the Raspberry hardware what to do. Linux is perfect for this.

- OpenCV: OpenCV is an Image Processing library created by Intel and maintained by Willow Garage. Available for $\mathrm{C}, \mathrm{C}++$ and Python.

- Python: Programming language used.

- Linux: Linux is the best suited OS for the raspberry pi.

\section{SYSTEM DESIGN}

A. Design overiew.

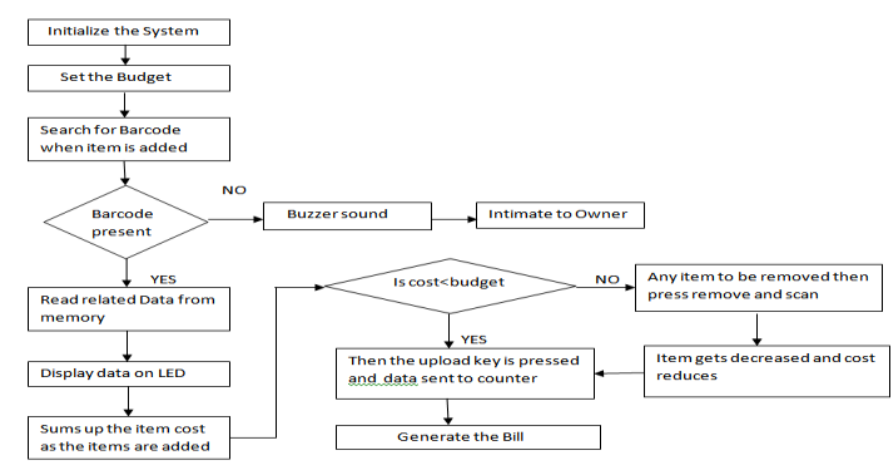

Figure 1. System Design 
B. System Architecture.

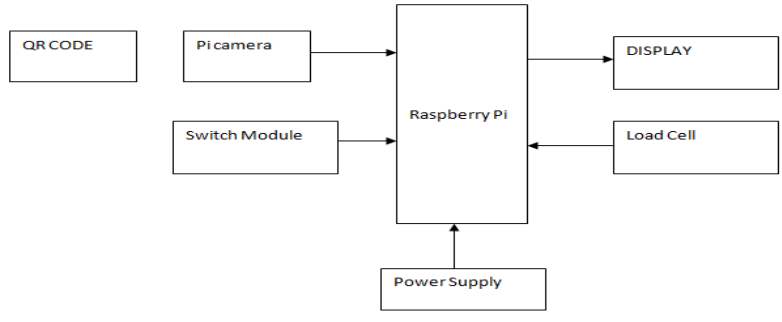

Figure 2. System Architecture.

Figure 2. shows the system architecture design and the working is listed below:

- Every product has a barcode which contains a Unique ID. These ID's are fed in the database assigned to the corresponding products [2]

- Load cell detects object weight put in the trolley and at the same time camera scans the barcode on the item.

- If any item is dropped into the trolley without scanning then an alarm with a beep sound is activated.

- The information of the product is extracted and displayed on the LCD screen. At the same time billing information is also updated

- When a customer wants to remove any product from the trolley, then that product needs to be scanned again.

- At the same time the billing information is updated. The total amount of purchases is also displayed on screen.

- These steps are repeated until the end of shopping button or send bill button is pressed. This generated bill is sent to billing side computer to get the computerized bill [2]

- Communication between PC and raspberry Pi is achieved through Wi-Fi [3]

- The customer can straight away pay the bill and leave [3]

- Inventory status of the products is also updated at the end of shopping. Simultaneously the temporary data present in microcontroller is reset, so that it can be reused.

- If the customer has registered user card, the payment can be done by swapping user card in the trolley itself.

- $\quad$ Payment is accepted and the customer can checkout.

\section{C. . Dataflow Diagrams.}

1. Dataflow Diagram - Level 0

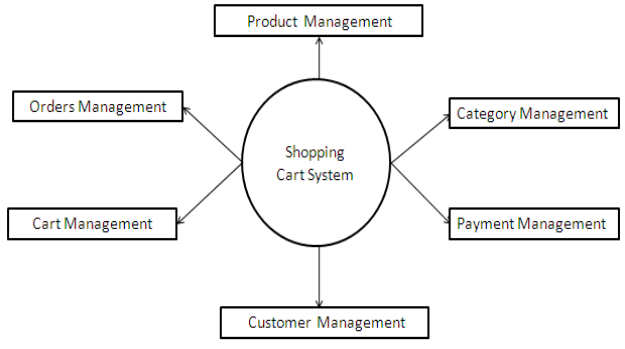

Figure 3. DFD-Level 0.

Figure .3 is the zero level diagram of shopping cart system. It's a basic level overview of whole shopping cart system or process being analyzed or modeled. It's designed to be an at-aglance view of cart, orders and login showing the system as a single high-level process, with its relationship to external entities of Products, Category and payments.

Level 0 process flow of shopping cart system:

- Managing all the products

- Managing all the category

- Managing all the payments

- Managing all the customer

- Managing all the cart

- Managing all the orders

2. Data Flow Diagram - Level 1

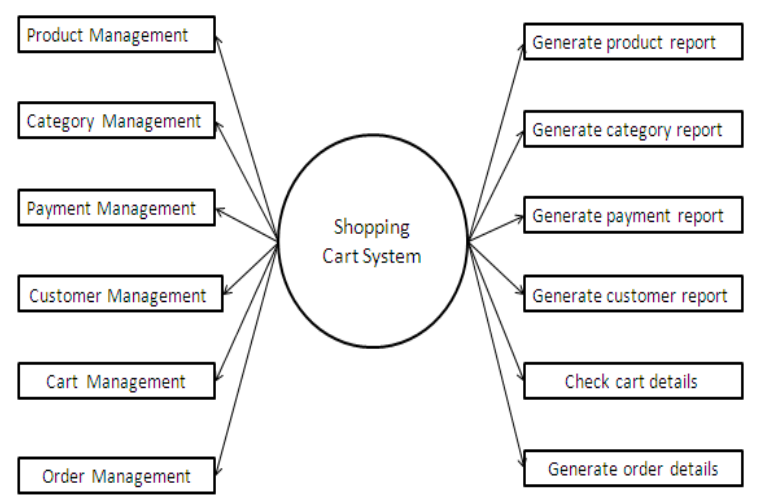

Figure 4. DFD-Level 1.

Figure .4 is the first level diagram of shopping cart system shows how the system is divided into processes, each of which deals with one or more of the data flows to or from an external agent, and which together provide all of the functionality of the shopping cart system as a whole. It also identifies internal data stores of orders, cart, customer, payments that must be present in order for the shopping system to do its job, and shows data flow between various parts of products, payments, orders, cart of the system.

Level 1 process flow of shopping cart system :

- Processing products records and generate report of all products

- Processing category records and generate report of all category 
- Processing payments records and generate report of all payments

- Processing customer records and generate report of all customers

- Processing cart records and generate report of all cart

- Processing orders records and generate report of all orders

3. Data Flow Diagram - Level 2

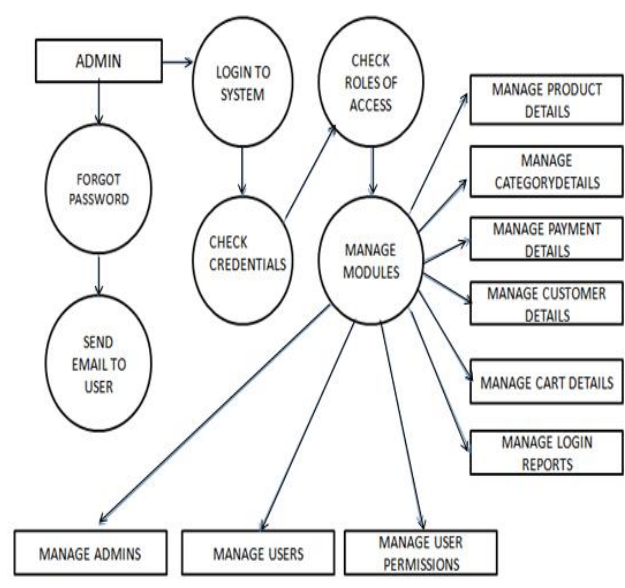

Figure 5. DFD-Level 2.

Figure .5 is the level $2 \mathrm{dfd}$ of the shopping cart system. It goes one step deeper into parts of level 1 of shopping cart system. It will contain more functionalities and details of shopping cart.

Level 2 process flow of shopping cart system :

- Admin logins to the system and manages all functionalities of shopping cart.

- Admin can add, edit, delete and view the records of products, payments, cart, and login

- Admin can manage all the details of category, customers and orders.

\section{CONCLUSION}

On a concluding note, the planned model is straightforward to use, low-cost and doesn't need any special coaching. This project part consists of the literature survey done on all the documented papers and therefore the info extracted from it. Functional, non-functional, software package and hardware needs have additionally been mentioned. The system style, system design, information flow diagrams (level zero, level 1, level 2), activity diagrams and sequence diagrams are shown. The more implementation of the project are going to be done very shortly

\section{REFERENCES}

[1]. C.Ezhilazhagan, R. Adithya, Y.L. Burhanuddin and F. Charles, "Automatic product detection and smart billing for shopping using Li-Fi," 2016 IEEE International Conference on Recent Trends in Electronics, Information \& Communication Technology (RTEICT), Bangalore, 2016, pp. 1723-1726.

[2]. J.C.N. Swamy, D. Seshachalam and S. U. Shariff, "Smart RFID based Interactive Kiosk cart using wireless sensor node," 2016 International Conference on Computation System and Information Technology for Sustainable Solutions (CSITSS), Bangalore, 2016, pp. 459-464.

[3]. Y. Berdaliyev and A. P. James, "RFID-Cloud smart cart system," 2016 International Conference on Advances in Computing, Communications and Informatics (ICACCI), Jaipur, 2016, pp. 2346-2352.

[4]. H. H.Chiang et al., "Development of smart shopping carts with customer oriented service," 2016 International Conference on System Science and Engineering (ICSSE), Puli, 2016, pp. 1-2.

[5]. Y. C. Wang and C. C. Yang, "3S-cart: A Lightweight, Interactive SensorBased Cart for Smart Shopping in Supermarkets," in IEEE Sensors Journal, vol. 16, no. 17, pp. 6774-6781, Sept.1, 2016.

[6]. P. Chandrasekar and T. Sangeetha,"Smart shopping cart with automatic billing system through RFID and ZigBee,"International Conference on Information Communication and Embedded Systems (ICICES2014), Chennai, 2014, pp.1-4.

[7]. Z. Pei, Y. Li and S. Xu, "An anchor-free localization algorithm for shopping carts on supermarket Internet of Things," Proceedings of 2012 2nd International Conference on Computer Science and Network Technology, Changchun, 2012, pp. 1401-1404

[8]. Rhee, Sokwoo, Deva Seetharam, and Sheng Liu. "Techniques for minimizing power consumption in low datarate wireless sensor networks." In Wireless Communications and Networking Conference, 2004. WCNC. 2004 IEEE, vol. 3, pp. 1727-1731. IEEE, 2004.

[9]. Thattai, Kuthsav, K. Bala Manikanta, Shiwangi Chhawchharia, and R. Marimuthu. "ZigBee and ATmega32 based wireless digital control and monitoring system For LED lighting." In Information Communicationand Embedded Systems (ICICES), 2013 International Conference on, pp. 878-881. IEEE, 2013.

[10].Apoorva Batra, Revanth Kamaraj and Marimuthu R, Solar Powered Full Bridge FET SMPS based Pulse Battery Charger with Power Management Using Atmega328, International Journal of Engineering and Technology, 5 (2), pp. 1672-1677. 2013.

\section{ACKNOWLEDGEMENT}

This project cordially acknowledges the college Global Academy of Technology and the Principal Dr.RanaPratap Reddy for providing this opportunity to build this project. Also a sincere acknowledgement to the Head of Department of Computer Science and Engineering Dr.Kavitha.C and the guide Mrs.SnigdhaSen for their immense support. 